\title{
De la historia «en migajas» a los objetos sólidos. Nuevos retos conceptuales, teóricos y metodológicos sobre Familia y ciencias sociales
}

\author{
Francisco Chacón Jiménez \\ Universidad de Murcia
}

\author{
FROM HISTORY IN «CRUMBS» TO SOLID OBJECTS. NEW \\ CONCEPTUAL, THEORETICAL AND METHODOLOGICAL \\ CHALLENGES ON FAMILY AND SOCIAL SCIENCES
}

Resumen

La reivindicación de los objetos como protagonistas explicativos del análisis histórico frente a la consideración de fragmentos tras el clásico, pero acertado para su contexto y momento histórico: L'Histoire en miettes: des Annales à la «nouvelle histoire», de François Dosse (1987), pretende ofrecer un giro teórico y epistemológico respecto al enfoque historiográfico. Para ello partimos del objeto Familia y lo situamos entre historia y ciencias sociales. Es, precisamente, en la creación del objeto, en las nuevas categorías analíticas y en la renovación de los métodos, donde se produce la verdadera síntesis en tanto que aportación y creación de conocimiento respecto a la organización social y sus procesos de funcionamiento.

El sistema de relaciones sociales y la integración de los individuos en la comunidad, le otorga a las familias el protagonismo en el cambio social o en las resistencias. Se determinan así factores como jerarquía, genealogías intergeneracionales, dominación, desigualdad y dependencia.

Palabras clave

Familia; Epistemología; Teoría; Objetos históricos; Ciencias Sociales

Códigos JEL: B50, D13, J12, N01

\section{Abstract}

This work examines the claim of objects as explanatory protagonists of historical analysis against the perspective set forth in the book L'Histoire en miettes: des Annales à la «nouvelle histoire», by François Dosse (1987). This article aims to study the theoretical and epistemological turn regarding the historiographical approach. With this objective, we start from the family as an object of study, so that we place it between history and social sciences. It is precisely in the creation of the object, in the new analytical categories and in the renewal of the methods, where the true synthesis occurs as a contribution and creation of knowledge regarding the social organization and its operating processes.

The system of social relations and the integration of individuals in the community provide families with the lead role in social change or resistance. Thus, factors such as hierarchy, intergenerational genealogies, domination, inequality and dependency are determined.

\section{Keywords}

Family; Epistemology; Theory; Historical objects; Social Sciences

JEL codes: B50, D13, J12, N01

Fecha de recepción del original: 25 de octubre de 2021; versión definitiva: 18 de diciembre de 2021.

Francisco Chacón Jiménez, Universidad de Murcia, Departamento de Historia Moderna, Contemporánea, de América y del Pensamiento y Movimientos Sociales y Políticos, Campus de la Merced, C/ Santo Cristo, 1, 30001 Murcia.

Tel.: + 34 868-883254 y +34 650114042; E-mail: chaconmu@um.es; ORCID ID: https://orcid.org/ 0000-0002-1900-9238. 


\title{
De la historia «en migajas» a los objetos sólidos. Nuevos retos conceptuales, teóricos y metodológicos sobre Familia y ciencias sociales ${ }^{1}$
}

\author{
Francisco Chacón Jiménez \\ Universidad de Murcia
}

\section{Presentación ${ }^{2}$}

Los historiadores hemos tenido que afrontar el hecho de que el conjunto de las ciencias sociales, desde la economía hasta la psicología, se han desarrollado en el contexto de la sociedad urbana e industrial del occidente contemporáneo con una grave consecuencia: no se le ha prestado la suficiente atención al análisis y procesos de evolución que han seguido las distintas culturas y formas del sistema social (Rowland, 1983: 83). Es un problema general y común que afecta al estudio de las instituciones y de la organización social, pues al formar parte y encontrarnos implicados directamente en ellas resulta difícil abstraernos del presente para pensar en términos de proceso. Y como consecuencia, la visión del mundo nos resulta difícil de analizar y explicar como una evolución con sus momentos y situaciones de cambio, resistencias y contradicciones.

La familia no es una excepción sino, más bien, una confirmación de dicha realidad. Por otra parte, de todas las instituciones sociales, la familia es la que exige una mayor contextualización científico-social. Su misma definición, desde una perspectiva comparativa es, inevitablemente, contextual. Por ello, la relación entre el análisis histórico y actual de las familias y las ciencias sociales se convierte en un objetivo prioritario para comprender la organización social y su proceso evolutivo. En palabras de Robert Rowland (1989), nos encontramos ante una historia multifacética, abierta a la demografía y a la antropología de la organización social de la reproducción.

Ya en 2009 se elaboró una reflexión conceptual (Chacón Jiménez, 2009: 133) que se traslada a esta presentación para, desde ella, abordar la articulación y la relación entre

\footnotetext{
1 Este estudio se integra y forma parte de los proyectos de investigación: “Entornos sociales de cambio. Nuevas solidaridades y ruptura de jerarquías (siglos XVI-XX)", con referencia HAR2017-84226-C6-1P, financiado por el Ministerio de Ciencia, Innovación y Universidades del gobierno de España, junto al proyecto: "Generaciones inciertas. Las familias de los influyentes españoles en tiempos de transformación (1740-1900)", con referencia PID2020-1135096B-100, financiado por el Ministerio de Ciencia e Investigación del gobierno de España.

2 El presente texto es una adaptación y puesta al día teórica y conceptual sobre el problema de la relación heurística, metodológica, conceptual y epistemológica entre historia y ciencias sociales, a partir del eje articulador que significan las familias. Basado en las reflexiones contenidas en Chacón Jiménez (2003) y en el reciente texto Chacón Jiménez (2020).
}

historia de la familia y ciencias sociales. Pero, previamente, situemos el objeto familia en su lugar epistemológico. Entre interdisciplinariedad versus desdisciplinariedad ${ }^{3}$, la multiplicación de los objetos de estudio sitúa a la historia en una encrucijada de difícil resolución. Por una parte, la profundización mediante miradas diversas y plurales en cada objeto: género, familia, grupos sociales, pobreza, esclavitud, estado, organización social, dificulta la verdadera interdisciplinariedad. Entendiendo ésta como la multiplicidad de facetas en sus espacios contextuales y culturales que explican el conjunto de la organización social. El paso fundamental que debemos dar, epistemológica y metodológicamente, es considerar los objetos como el verdadero eje de articulación y explicación histórica. La fortaleza a la vez que la gran amplitud de perspectivas que cada uno de ellos ofrece permite recoger, verdaderamente, el sentido último del análisis histórico. Solo el despliegue de líneas, perspectivas, miradas, relaciones $y$, sobre todo, preguntas que cada objeto puede plantear a unas fuentes ampliadas, muy especialmente en el caso del objeto familia, puede dar la verdadera respuesta y dimensión dialéctica interdisciplinariedad versus desdisciplinariedad. Nuestra apuesta es, decididamente, diferente pero innovadora respecto al clásico y ya manido problema. Es necesario erigir el objeto, el sólido objeto, en el protagonista. Y a través de un recorrido relacional entre historia y ciencias sociales concederle a familia, desde esta nueva óptica, su verdadera dimensión y alcance explicativo al conjunto de la organización social.

Partimos de entender la familia como una manera de pensar; es decir, como una práctica o un hábitus más que una estructura formal. Vivimos mentalmente la familia, nos expresamos en términos de familia y todo ello a pesar de vivir en una sociedad caracterizada por lo individual. La realidad institucional y su marco jurídico queda reflejada de forma expresa y precisa en el derecho civil y el derecho de familia, aunque la desinstitucionalización es una condición propia de la realidad social actual. La familia adopta unas formas que cambian y se proyectan a través de los nacimientos, defunciones y uniones. La familia es también una representación social expresada mediante

\footnotetext{
3 En una reflexión de Chacón Jiménez (1991) se señalaba que el resultado de una aproximación interdisciplinar "puede ser el de una mezcla o superposición de materias que no aporta prácticamente casi nada". Joan Bestard ya planteó en un sugerente articulo la necesidad epistemológica de intentar resolver el difícil binomio interdisciplinariedad versus desdisciplinariedad (Bestard Camps, 1980: 154-162).
} 
una descripción que recoge la morfología y sus funciones. Pero, lo más destacado es que nos encontramos ante una articulación entre el orden biológico y el sistema social. La Etnografía y la Antropología han enseñado a considerar la familia como una construcción cultural y como la unidad elemental de la función social. La familia es, pues, un sistema de relación entre el contexto social y la red de relaciones comunitarias.

La solidaridad familiar, que desde la crisis de inicios de la década de los setenta y de los años ochenta del siglo XX se ha percibido con claridad en la sociedad española y convirtió a la familia en el colchón de apoyo que impidió tensiones sociales, no es una estrategia coyuntural como respuesta a una situación determinada, sino que responde a una tradición y a unas prácticas culturales que tienen a la familia como punto de apoyo y referencia en la realidad diaria y en la vida cotidiana. Este profundo familismo de la sociedad española, que hunde sus raíces en la fuerte oposición a los principios de la Reforma protestante, y que se concreta en los discursos de limpieza de sangre y su profundo significado social y cultural (Hernández Franco, 2011 y 1996), así como su permanencia en el tiempo, hace que el Estado y las instituciones le presten una escasa atención. Lo cual reafirma la trascendencia de la institución familiar como objeto de análisis, tanto en el plano individual de cada familia como desde el parentesco y las relaciones que se establecen en las distintas unidades familiares, y en la proyección de un conjunto de familias unidas por razones sociales, económicas, políticas o de clase.

Conocer y explicar la evolución y el proceso seguido hasta el momento presente, le otorga a la historia de la familia una dimensión que exige la prudencia propia de la contextualización histórica para alejarse del presentismo fácil, pero también la posibilidad de analizar cómo y a través de qué mecanismos se han desarrollado complejos procesos de reproducción, movilidad social y cambio histórico en la organización social y su influencia sobre la familia. El acceso de la mujer al trabajo, el envejecimiento de la población y el consecuente cuidado de los ancianos, atribuido por hábito y costumbre a las hijas, han transformado desde los años ochenta del siglo XX las pautas de conducta y sociabilidad desde la familia y respecto a la familia. Sin embargo, la tradición sigue teniendo fuerza. Pero se ha roto en las últimas décadas con las generaciones que se incorporan al mercado, al matrimonio y a las posibilidades de tener hijos desde posturas de independencia, autonomía y, sobre todo, de individualidad. El grupo familiar no ha dejado de ser un punto de apoyo en momentos de dificultades; todas las estadísticas europeas sobre la ausencia de los hijos del hogar o la consideración de la familia respecto a la preocupación que sobre estos temas tienen los jóvenes, colocan en un lugar muy destacado la consideración de la familia como institución y espacio social básico, lo que explica la compleja y contradictoria situación presente. Contradicción que explica porqué a pesar de la importancia de la familia en la sociedad ésta no se haya tratado como objeto científico hasta fechas tardías.

Una parte fundamental de la renovación teórica y epistemológica ha girado alrededor de la creación de nuevos objetos de investigación. La dimensión interdisciplinar ha convertido en aportaciones autónomas lo que en prin- cipio eran nuevos objetos fruto de la relación entre historia y ciencias sociales. Ello ha producido una situación de inestabilidad teórica y metodológica. La situación ha transcurrido, en nuestra opinión, de la siguiente manera: algunos objetos, como mujer, familia, estado, grupos sociales, han significado una verdadera revolución ya que han alcanzado un desarrollo propio que les ha permitido, además, establecer sólidos lazos de relación interdisciplinar e integrar en el análisis histórico categorías y métodos procedentes de otras ciencias sociales. Especialmente al adoptar el método nominativo frente al agregativo, con la enorme trascendencia que tiene respecto a la utilización interrelacionada de fuentes prosopográfico-biográficas, bases de datos, reconstitución de parroquias en lugar de reconstitución de familias mediante cruces de fuentes, o network analysis. Lo que ha significado en muchos casos una revisión de las fuentes e incorporación de otras.

Simultáneamente, y a la vez que se producían estos significativos avances, se revisaban antiguas líneas de investigación; tal vez la más significativa haya sido la denominada tradicionalmente como historia política. A partir de aquí, y desde luego hace algún tiempo, comienzan a levantarse voces reclamando una necesaria integración en una historia global y general. Sin embargo, no parece que el tiempo de las síntesis haya llegado, ni siquiera que este próximo. Por otra parte, el problema ni es tan sencillo ni tan simple y debe situarse en otro plano: la necesaria tensión y debate permanente entre macro-micro, general-particular o universal-local se ha visto superada, ampliamente, por la profundidad, densidad y complejidad de la ciencia histórica de una nueva etapa caracterizada por la comprensión del sistema de relaciones sociales que explica la manera y el modo en que se encuentra organizada la sociedad,

Dos importantes aportaciones teóricas han contribuido a potenciar esta perspectiva que relega a un plano secundario la elaboración de síntesis; al menos aquellas que se entiendan como volver a escribir una historia general con las conclusiones obtenidas a partir de los nuevos objetos e incluyendo un epígrafe o apartado sobre los mismos. El problema es mucho más complejo, y el proceso de interdisciplinariedad no solo ha propiciado nuevos objetos y con ello métodos y nuevas categorías analíticas, sino también una reinterpretación del proceso histórico que no puede ser analizado como una mera agregación o superposición, sino como una nueva y novedosa aportación. De todas formas, la síntesis llegará, pero bajo esta óptica y desde esta concepción.

Es aquí, es decir, en la creación del objeto, en las nuevas categorías analíticas y en la renovación de los métodos, donde se produce la verdadera síntesis en tanto que aportación y creación de conocimiento respecto a la organización social y sus procesos explicativos. El resultado es siempre una mayor complejidad, que en una primera etapa apareció como la desintegración en "migajas" (L Histoire en miettes, de Françoise Dosse) de la historia; y después, como la necesidad urgente de realizar síntesis que lograsen evitar que la historia derivase en el Sherlock Holmes de los microhistoriadores. Enfoques, que sin ser erróneos, no alcanzan ni superan la etapa primaria del proceso interdisciplinar. Algunos autores han llegado a plantear la necesidad de dirigir las investigaciones hacia la desdisciplinarie- 
dad como objetivo clarificador de cada ciencia (Bestard i Camps, 1980: 154-162). No se trata de una vuelta atrás, por cuanto consideramos que el camino recorrido es irreversible. Se trata más bien, en nuestra opinión, de un importante avance teórico de Joan Bestard. Lo cual nos permite dar un paso adelante al sugerir la solidez de los objetos como propuesta de solución teórico-metodológica y epistemológica. Por lo que es necesario insistir en la necesidad de proseguir ampliando las enormes posibilidades que ofrecen los objetos en su perspectiva inter y multidisciplinar dentro de las ciencias, en general, y muy especialmente las sociales.

El problema es el de ajustar los métodos adoptados de otras ciencias sociales a las categorías y a las fuentes de cada disciplina, considerando que en el caso de la historia, el tiempo, el contexto y las diferencias espaciales y de sistema social en cada periodo, obligan a una rígida y profunda critica de fuentes y de métodos. Como indicaba Gerard Delille, el historiador no puede ser el antropólogo del siglo XV, XVI, XVII, o XVIII. Lo mismo se podría afirmar de las restantes ciencias sociales. Sin embargo, las categorías analíticas y conceptos como reproducción social o movilidad social, o métodos como microhistoria, redes sociales, han potenciado de una forma exponencial, en sentido comprensivo y explicativo, el análisis histórico.

Una de las aportaciones más notables es la que ha permitido la integración del individuo en los distintos espacios jurídicos, políticos y de sociabilidad, comenzando, precisamente, por la familia y después: casa, parroquia, gremio, cofradía, concejo y, en general, la comunidad. Evidentemente, cada una de estas entidades y realidades sociopolíticas presenta y permite, desde la óptica de la familia, una serie de posibilidades analíticas amplias en tanto que conceptos como ciclo de vida, trayectoria o estrategia, forman parte del análisis que, a partir del método nominativo y al integrar el objeto familia en la necesaria explicación de la organización social, le otorgan una dimensión temporal y genealógica vertical a la vez que horizontal desde el punto de vista relacional entre los individuos de una comunidad.

Es por ello por lo que vamos a articular nuestro análisis a partir de dos bloques: la relación entre historia y ciencias sociales $y$, en segundo lugar, estudiando los individuos en su relación con la comunidad en la que viven y se desarrollan.

\section{Historia y ciencias sociales}

El surgimiento de nuevos enfoques y planteamientos en el horizonte historiográfico sitúa a la familia y su capacidad explicativa en un primer plano. Es importante especificar cómo desde cada disciplina la familia ha tenido su propia evolución. No se trata ni pretendemos llevar a cabo un recorrido temporal del proceso de relación entre historia de la familia y otras ciencias sociales, lo que nos interesa es la manera en que ha sido integrado el concepto y la realidad familiar en el análisis histórico; es decir, qué factores y elementos propios de cada disciplina han ido formando parte del análisis y del objeto familia, y de qué manera se han integrado en la evolución histórica. Proceso que ha tenido varias fases; la primera ha sido la de una superposición casi caótica y que solo agregaba métodos pero casi nunca análisis; otra más depurada en la que el historiador ha conocido y estudiado los métodos de trabajo de otras disciplinas para aplicarlos a la investigación histórica dando lugar, entonces, a una adaptación más refinada metodológicamente a la vez que sugerente.

Pero antes hagamos un recorrido por el tiempo histórico de la mano de Tamara Hareven, cuya investigación se publicaba por primera vez en $1991^{4}$ :

"Investigaciones históricas recientes han cambiado nuestro concepto de la vida familiar en el pasado y han obligado a revisar algunas de las generalizaciones sobre el impacto de los grandes procesos de cambio social en la familia y la sociedad. El estudio de la historia de la familia tiene profundas y complejas raíces en la demografía histórica de principios de los años sesenta del siglo veinte, así como en la llamada "nueva historia social" de la misma época; en concreto en EEUU. Ambas se han encargado de revisar lo que sabemos de los modos de vida de la población y gente común a la que se ha pasado a considerar como sujeto, tanto activo como pasivo del proceso de cambio" (Hareven, 1995: 101).

Con estas seminales palabras, escritas hace treinta años, se explicaban, sintetizaban e integraban los avances historiográficos (vigencia plurisecular de la familia nuclear y división geográfica en función de la relación entre edad al matrimonio, herencia y estructura del hogar) llevados a cabo por el Cambridge Group (Laslett, 1972) desde su fundación en 1969; y que continuaron a lo largo de los años setenta y ochenta del siglo XX.

La unión de demografía histórica con la entonces llamada nueva historia social, ha abierto dos vías de investigación que han renovado por completo los objetivos explicativos de la evolución y procesos de cambio o/y resistencias de las sociedades en el período comprendido circa mitad siglo XVIII y el tiempo actual: revisar los modos de vida de la población y que ésta, a través de las familias, se convierta en sujeto del cambio social. A los historiadores y sociólogos que durante una generación se dedicaron al análisis de la geografía de las formas familiares, se les habría un nuevo campo de hipótesis y preguntas con las que interrogarse sobre el impacto de los grandes procesos políticos y jurídicos de cambio social: Revolución Francesa, Revolución industrial, promulgación de constituciones y de códigos civiles durante la segunda mitad del siglo XVIII y el XIX, a través de las transformaciones, permanencias o/y continuidades que experimentan las familias. Éstas han sido, pues, las verdaderas protagonistas del proceso de cambio social.

\footnotetext{
${ }^{4}$ El artículo apareció por primera vez en 1991 en American Historical Review, dando permiso para su traducción y publicándose en 1995 en la referencia bibliográfica que aquí se señala.
} 
Desde esta óptica y con este razonamiento se le concede carta de naturaleza a dos problemas fundamentales:

a) La consideración de interdisciplinariedad en el estudio y análisis de las familias pero desde la óptica de objeto social.

b) La ruptura de los clásicos períodos y etapas históricas de raíz académica entendidos, en muchas ocasiones, como espacios compactos, cerrados e incomunicados $y$, sin embargo, precisados de un necesario continuum temporal para su comprensión y explicación. Así, pues, los retos a los que se enfrenta la historiografía se han centrado en tratar de comprender y explicar permanencias, continuidades o/y cambios sociales que tienen lugar a partir de hechos históricos concretos que implican aprobación de normas legislativas por impulso e iniciativa de diferentes grupos sociales, en el interior de un determinado marco jurídico-político. En consecuencia, el estudio, análisis y comprensión de las realidades cotidianas se lleva a cabo desde un proceso histórico caracterizado y definido por esta compleja, diversa, plural y multicausal realidad.

Expliquemos esta complejidad refiriéndonos, en primer lugar, a la demografía histórica y la historia de la familia. Se han desarrollado sin tenerse en cuenta la una de la otra. La primera implica no a los individuos sino a poblaciones enteras. $Y$ al definir la estructura y la dinámica de éstas se ve obligada a dejar de lado un cierto número de factores que definen el sentido histórico y sociológico de los comportamientos demográficos, y que permiten enunciar la naturaleza de la relación entre dichos comportamientos y sus contextos socioeconómicos y culturales. Por otro lado, muchos historiadores de la familia conducen sus investigaciones y definen su objeto de estudio de un modo que, implícita o explícitamente, hace abstracción de su representatividad. Este problema y el de compresión de los factores económico-sociales y de raíz cultural que implican la dinámica de las poblaciones es de origen y solución relacional; y ésta depende de la capacidad que tengan los científicos sociales para desarrollar conceptos estructurantes que especifiquen la naturaleza de las múltiples relaciones e interacciones entre sociología y el sistema demográfico y su contextualización.

De la mano de la sociología, la familia comienza a ser tenida en cuenta a finales del siglo XIX y principios del XX. Tras las aportaciones de Durkheim o Le Play se comienzan a deshacer los mitos sobre la pervivencia de la familia tradicional y la ruptura que originaron los procesos de industrialización, urbanización y migración. Pero la sociología privilegia el análisis de las funciones, por lo que su interés se dirige a precisar el cambio y la pérdida progresiva de éstas en una evolución histórica. Con esta orientación la contribución de la sociología ha parecido limitada, ya que su carácter finalista con la progresiva pérdida de funciones produce un aislamiento teórico. Sin embargo, se ha incorporado a los estudios de familia en una triple vertiente: a partir de profundizar en aquellos factores que explican el distinto tipo de funciones económicas: consumo, producción, herencia, transmisión, mercado y otras en relación con las familias; o demográficas; o bien sociales: formas de organización, constitución de grupos.

La influencia de la sociología ha derivado a un segundo plano de mucho mayor interés: me refiero al método de la sociología funcionalista norteamericana de los años cincuenta: network analysis, y a sus derivaciones sobre los procesos de movilidad social aplicados y proyectados a la realidad familia; que permiten considerar los conceptos de estrategia o ciclo de vida, que no podríamos entender como adscritos a una influencia exclusivamente sociológica. Tanto la demográfica histórica como un enfoque genealógico de orientación demográfica y social permiten avanzar, profundamente, en el conocimiento de los procesos que protagonizan las familias; pero siempre considerando los contextos culturales y valores sociales.

Respecto al sistema demográfico, en ocasiones se ha intentado buscar respuestas sobre comportamientos domésticos. La imposibilidad es estructural en tanto que estos comportamientos se refieren a individuos y a grupos sociales concretos, Un análisis demográfico o de estructura del hogar implica a la totalidad de una población que tendrá un carácter heterogéneo y diverso. Porque la pregunta clave es: cómo se forman y crean los destinos individuales y en qué medida son influenciados por las relaciones sociales. Indiquemos los ya conocidos métodos de análisis que desde mitad del siglo XX han puesto de relieve la trascendencia de las familias en relación con el comportamiento individual. En primer lugar, el método de reconstrucción de familias Henry-Fleury (1956) mediante el cruzamiento nominativo; segundo, lo que supone la obra de Philippe Ariès; no tanto su conocido libro sobre el niño y la vida familiar en la sociedad del Antiguo Régimen (Ariès, 1960), como su trabajo respecto a las actitudes ante la vida (1948); y en tercer lugar, el Grupo de Cambridge. Creado en 1964 por P. Lastlet y A. Wrigley, produce la más influyente de las aportaciones metodológicas a partir del congreso de $1969^{5}$ y su posterior publicación: Household and Family in Past Time, 1972. Es decir, entre 1956 y 1972 y la publicación de Des registres paroissiaux a l'hitorie de la population. Manuel de dépoullement et d'exploitation de l'étar civil ancien (1956) y Household and family in Past Time (1972), transcurren dieciseis años durante los cuales se sientan las bases de la historia de la familia y de los instrumentos teóricos que significan la renovación de la demografía histórica y los intentos de acercamiento teórico y metodológico a los procesos de evolución y cambio de las poblaciones en su conjunto y de sus unidades familiares e individuales.

En 1981 se sumó la back-proyection con la utilización de series de nacimientos y defunciones para conocer la estructura de la población en épocas anteriores. Se concretó en el monumental trabajo de E.A. Wrigley y R.S. Schofield (1981), The population History of England, 1541-1871. Que fue completado con los debates y discusiones suscitadas en el volumen dedicado al análisis de las variables interre-

\footnotetext{
5 Organizado desde el grupo de Cambridge con la participación activa de Isabel Moll por parte de la Universidad de las Islas Baleares, se celebra en Palma de Mallorca (noviembre 1989, coincidiendo con el veinte aniversario del encuentro de Cambridge), una reunión internacional: Family structure, life course and socioeconomic behaviour, con la presencia de Peter Laslett y destacados demógrafos, geógrafos, antropólogos, sociólogos, economistas e historiadores europeos.
} 
gionales en Europa, en lo que respecta a las estructuras familiares y su contexto socio-económico: R. Wall, J. Robin, P. Laslett, Family Forms in Historic Europe (1983). La dificultad se ha planteado durante mucho tiempo en la escasa utilidad que para la historia de la familia suponía la familia conyugal biológica. Solo cuando la identificación nominativa se ha convertido en el método de trabajo y el cruce de fuentes con las fichas de familia, a partir de la misma reconstitución de Henry-Fleury, se ha producido una verdadera renovación teórica.

Sin embargo, es posible llevar a cabo y deducir determinado tipo de comportamiento si, por ejemplo, desagregamos los datos de una población concreta según grupos de edad, actividad del cabeza de familia y los relacionamos con el tamaño y la estructura del hogar, el número de hijos y la mencionada actividad (Chacón Jiménez et al, 1986 y Chacón Jiménez, 1987). En cualquier caso, si quisiéramos profundizar, conocer y comprender qué elementos culturales explican determinadas funciones y decisiones es el contexto, en sentido amplio y con plena incorporación del análisis histórico, el que puede llegar a deducir y explicar dicha realidad ${ }^{6}$. Estas perspectivas han superado, ampliamente, los orígenes de los estudios de familia y de demografía histórica.

Coincidiendo con esta etapa (1956-1983), asistimos a notables avances historiográficos que se concretan en intentos de modelización a través de establecer relaciones entre tipología y variables demográficas y económicas (edad femenina en las primeras nupcias, niveles de celibato definitivo, sistemas de herencia). Posteriormente, aparecen nuevos problemas en el horizonte: 1) integrar el parentesco en su dimensión social; 2) analizar y explicar los vínculos que ponen en relación a los individuos, o 3) situar a la familia en la red social de solidaridad, relaciones de dependencia y ciclo de vida. El parentesco, y en su contexto la Antropología, se convierte en el verdadero cemento de estas tres incompletas, pero básicas, miradas; junto con la creación a finales de los años setenta del siglo XX del método nominativo y la microhistoria?.

Además, no se debe plantear de manera aislada la relación histórica de familia-ciencia sociales. Nos referirnos al matrimonio, la variable mas sociológica de todas las demográficas y en la que cristalizan estrategias sociales de alianza y parentesco; transferencias económicas y de propiedad y, por supuesto, de poder. Precisamente, la antropología aporta a la historia de la familia uno de los razonamientos y explicaciones fundamentales para la comprensión del proceso histórico. Si anteriormente señalábamos el periodo 1956-1983 como clave en la relación historia de la familia-ciencias sociales, al hablar de antropología tenemos que remontarnos a finales del siglo XIX. Los sistemas de filiación y las reglas del parentesco son la base sobre la que se levanta el complejo edificio de cualquier organización social.

La puesta en marcha de los mecanismos de la alianza se configura alrededor del matrimonio, y es aquí donde

\footnotetext{
$\overline{6}$ Un estudio referido al contexto histórico entre el siglo XVIII y el momento presente se aborda en Chacón, 2020.

7 Sobre la microhistoria fueron relevantes las propuestas de Ginzburg y Poni, 1979.
}

la relación con la antropología alcanza sus máximas posibilidades. El parentesco, en palabras de Joan Bestard, es un vehículo de relación y solidaridad social que implica no separar la corresidencia del conjunto de relaciones de parentesco (Bestard, 2001). Precisamente, es el parentes$\mathrm{Co}^{8}$ el que otorga su pleno sentido a la idea de familia. Se supera los límites del hogar, y es la alianza y los vínculos y lazos familiares los que operan. Las relaciones de parentesco intervienen activamente en los procesos históricos de formación de grupos sociales, y han jugado un papel muy activo en la conformación de clases sociales.

Gerard Delille plantea en una nota critica $(2001)^{9}$, el cambio de todos los mecanismos tradicionales del sistema social: especialmente, la separación hermano/hermana con destinos económicos y matrimoniales independientes, a lo que se une la proliferación de los matrimonios consanguíneos y cercanos con prácticas (sororato, levirato) habituales ahora (finales XIX, principios del XX) pero prohibidas, tradicionalmente por las normas de la iglesia. Su significado es fundamental: la cercanía del parentesco a través de matrimonios supuso la posibilidad de construcción de redes de relaciones que con la concentración de capitales daban lugar a defender intereses basados en dinastías familiares. Como, por otra parte, el proceso histórico ha dejado claro las estrategias de alianza y la relación clientelar y de patronazgo para acceder al control de los recursos, se crea una cultura y una práctica de relaciones familiares ${ }^{10}$. La sugerencia de Gerard Delille y de Joan Bestard, es la ruptura de este sistema a través de la explosión de consanguinidad y de los matrimonios cercanos que favorecen la conformación de las clases sociales. Explicación novedosa respecto a las definiciones tradicionales de orden político que, lógicamente, no se pueden separar de las estrategias familiares y económicas. Todo ello se configura alrededor de un primer círculo basado en las relaciones de consanguinidad. Que se mezclan, unen y confunden, en muchas ocasiones, con el originado por el parentesco ficticio y la alianza. Desde estos círculos, y a través de la amistad, se proyecta la relación familiar hacia otros grupos sociales que pueden convertirse en lazos clientelares de carácter dependiente y vertical.

El entramado que se produce tiene y presenta una gran complejidad por cuanto los lazos y vínculos de relación social (el segundo aspecto que pone en relación a los individuos) están atravesados por la consanguinidad, el parentesco, el parentesco ficticio, la alianza, el clientelismo $y$, además, se entretejen sobre diversas instituciones que actúan como órganos independientes. Y quienes les dan vida pueden y suelen estar relacionados entre sí por fuertes y sólidos lazos de sangre o/y amistad. Por ello, es fundamental reconstruir la red de relaciones que se entrelazan a partir de las familias y en las que los intereses horizontales

\footnotetext{
${ }^{8}$ Nuestras referencias se dirigen a destacar la relación entre Parentesco e historia. Ver al respecto la sugerente y completa reflexión de Porqueres (2015).

${ }^{9}$ Véase también el monográfico: Popolazione e Storia (2013), Dousset, Faggion, Minvielle y Regina (2013). Respecto a normas y regularización del parentesco, ver: Doyon, Odier da Cruz, Praz, y Steinberg (2013).

10 Una excelente síntesis y puesta al día de esta compleja pero básica y fundamental problemática en Delille (2017), especialmente el apartado VI: "Los bienes circulan, los hombres también", pp. 227-231; y el apartado II: "El mundo cristiano: entre guerras y matrimonios", pp. 264-271.
} 
de grupo están cohesionados por la familia y la amistad, mientras que la adhesión clientelar, al tener un carácter jerarquizado aparece unida por relaciones de dominación vertical. Pero no pensemos que ambas situaciones se dan en estado puro; al contrario, el camino de la relación o el del ascenso o descenso social suelen caracterizarse por un entramado a la vez vertical y horizontal, con nudos centrales muy fuertes que conforme nos alejamos de ellos, tanto en sentido vertical como horizontal, se van debilitando. Estas relaciones se plasman, se consolidan o se enfrentan alrededor de los poderes locales, señoriales, eclesiásticos o del Rey. Y solo desde una perspectiva generacional y de explicación genealógica es posible entender los ideales de reproducción y perpetuación. Esta es la causa y la explicación de la fuerte, y muy necesaria, presencia historiográfica de los estudios sobre redes sociales. Lo que nos plantea la relación:

\section{Individuo-comunidad}

A partir de aquí, nos parece necesario mostrar, por una parte, las distintas articulaciones y relaciones que en función de los distintos niveles sociales, espacios, contexto jurídico y etapa histórica concreta, explican comportamientos y diversidades sociales. De esta manera, se explican las distintas situaciones de cambio social teniendo en cuenta que se pueden producir cambios idénticos en contextos totalmente diferentes. Es importante detectar qué mecanismos producen estos cambios y a partir de qué grupos sociales y con qué estrategias, fines e intereses. Se presentan una serie de posibilidades analíticas amplias en tanto que conceptos como ciclo de la vida, trayectoria o estrategia, forman parte del análisis que, a partir del método nominativo y al integrar el objeto familia en la necesaria explicación de la organización social, le otorgan una dimensión temporal y genealógica entre los individuos de una comunidad.

El matrimonio se erige en un instrumento básico y fundamental. Investigar y analizar cuáles son los criterios sociales y económicos de elección de cónyuge y en qué momento y bajo qué circunstancias cambian, es una explicación que nos introduce en la conformación y configuración de las clases sociales. No se trata, en nuestra opinión, de un concepto y una realidad que exija esperar a las revoluciones burguesas y a la redacción y proclamación de las constituciones del siglo XIX para confirmar su existencia.

La homogeneidad de intereses y las relaciones de dominación, plasmadas en jerarquías, explicitan con claridad intereses bien diferenciados pero ocultos, a la vez que integrados en las relaciones clientelares y de patronazgo que establecen lazos y vínculos que pese a su verticalidad y dominio guardan el sabor de su origen feudal, en cuanto al carácter donativo mutuo de criado y señor. Es desde esta realidad, desde la que se configuran redes de carácter económico, social y de poder atravesadas y tejidas, lógicamente, por el parentesco, en las que los individuos, en defensa de los intereses de las familias aparecen como una articulación fundamental. Cuyo estudio exige desvelar las relaciones de poder, tanto en las comunidades locales como en las relaciones entre la monarquía y los poderes intermedios y locales. Es aquí donde tienen todo su sentido el levantamiento de genealogías sociales que desde una perspectiva antropológica, sociológica y demográfica permitan dos cosas fundamentales: primera, una identificación nominativa de los individuos insertos en su espacio: social, de actividad y de patrimonio; $y$, segunda, su integración en territorios de mayor dimensión y posibilidades analíticas. Me refiero a las redes; pero en sentido multirrelacional y con el objetivo de detectar los mecanismos de control, y dominio social y político.

Podemos señalar que la sociedad europea anterior a 1789 se configuraba y organizaba como estados, entendiendo por tales: distinciones y consideraciones culturales con proyección social y prácticas de privilegios y exenciones que se inician en el propio nacimiento $y$, por tanto, se heredan; los individuos organizados de esta forma se integraban en corporaciones que regulaban la vida política y social. Desde esta descripción, conceptos como reproducción social o movilidad social son contrastados a través, por ejemplo, del levantamiento de genealogías sociales que intentaran medir los procesos de dominio, dependencia y desigualdad. Pero la genealogía es algo más que un método, y en su parte conceptual se integran el ciclo de la vida, la trayectoria profesional de los individuos, que no se debe particularizar sino insertar en un contexto más amplio de familiares y de alianza; para entender, entonces, la trayectoria individual y, finalmente, la estrategia. Aquí se explicaría el concepto y las posibilidades teóricas que ofrecen, como método de análisis pero sin convertirlo en una explicación univoca, las redes sociales.

Hay que entender la familia como algo más que un fragmento de una historia social dividida. Pero nuestra obsesión será siempre la misma: comprender la organización social. Dos grandes sistemas de estratificación debemos de emplear: el basado en el linaje y el que tiene a la riqueza como criterio. Ahora bien, la historiografía nos ha indicado con gran claridad la importancia y trascendencia de las relaciones sociales, y la estrecha relación entre ambos. Pero la riqueza no hay que medirla en términos monetarios, con ser importante, sino de amistad y relación. Permitían obtener gracias y mercedes desde las que, finalmente, se conseguían provechosos recursos y bienes patrimoniales. Más que relación es superposición de ambos valores: recursos y relaciones; y ambos imprescindibles. Su escisión es un rasgo distintivo de la presencia de otros valores en la justificación y legitimidad del orden social. A partir de los heredados se adquiere un determinado status; con el que se sitúa a los individuos en la escala social. Individuos que no permanecen estáticos, y que en contextos específicos buscan y procuran para ellos y sus familias y para el grupo al que pertenecen, la promoción social.

El cómo, los medios y las estrategias es, precisamente, nuestro trabajo como historiadores. El status al que acabamos de referirnos, en la sociedad tradicional se hereda, mientras que se adquiere en la contemporánea. Ahora bien, este es uno de los cambios y transiciones que con 
cierto carácter finalista algunos autores plantean dentro de una explicación evolutiva de la familia afectada por procesos civilizatorios. Así, pasamos de una sociedad basada en los vínculos y lazos personales a otra basada en la riqueza y el poder que conlleva el dinero. Y de una sociedad basada en la casta y el patronazgo en la que a los hijos se les valora como hombres de armas y mantenedores de un grupo doméstico y familiar, a un mundo de mercado, venta de servicios y profesionalidad. Otros procesos se articulan alrededor de la separación entre sagrado-profano, público-privado y heredado-adquirido; en cualquier caso se trata de perspectivas generales que precisan estudios de caso para una comprobación empírica a la vez que integración en cada coyuntura y contexto, para lo cual hay que medir bien la escala de análisis que se utiliza y la revisión de fuentes y utilización de nuevas.

Integrando nuestro análisis histórico en las distintas ciencias sociales: etnografía, demografía, derecho civil, antropología, sociología, podríamos señalar dos grandes etapas:

1) Desacralización, en donde el poder civil a través de los códigos y la independencia de lo religioso permite iniciar una etapa que en España tiene a la ley de matrimonio de 1870 y el código civil de 1898, como precedentes de lo que será en 1981 (con la breve vigencia del contrato sobre el sacramento del matrimonio durante la II República, anticipándose en medio siglo) la ley de divorcio y en 2005 la ley de parejas de hecho. A esta etapa prosigue, sin solución de continuidad:

2) Desinstitucionalización, en la que nos encontramos actualmente y que se caracteriza por las uniones libres y el individualismo como valor social y cultural básico. Pese a ello, la fuerza de la tradición y la continuidad y adaptación en los valores familiares nos presentan una sociedad contradictoria: entre la fuerza de la razón familiar y la de las políticas públicas, que tiene como eje de equilibrio el papel de la mujer. Y que se ha modificado y cambiado notablemente ante las nuevas realidades de la diversidad de familias y pluriparentalidades e individualización y autonomía de las personas.

En este punto, España constituye un laboratorio especialmente significativo por la simultaneidad y convivencia entre el modelo tradicional de familia, sobre todo en ciertas prácticas y mantenimiento de lazos y vínculos entre generaciones, y las nuevas familias caracterizadas por la libertad de elección en la formación de la pareja, el principio de igualdad entre géneros, y la centralidad del niño en la formación de las relaciones familiares, así como el interés superior del menor como principio de doctrina jurídica (Chacón Martínez, 2019). Nos encontramos ante la contradicción que supone el progresivo proceso de privatización de la vida doméstica frente a la constante presencia pública de la familia como institución articuladora del sistema social.

Acercándonos a la conclusión planteada en el reciente congreso celebrado en Lisboa (enero 2020) sobre: "Familias, identidades y cambio social en España y Portugal. Siglos XIX-XXI, Perspectivas comparadas europeas", seña- laremos que una vez superada la fase de aislacionismo de cada disciplina científica y de histoire en miettes (consecuencia de la multiplicidad de objetos), nos encontramos en un contexto de explicación global y de larga duración en el que solo la síntesis, el fortalecimiento de los objetos y las miradas e hipótesis integradoras pueden captar el verdadero sentir y evolución de un proceso que necesita recurrir a un referente o elemento de definición (en este caso las familias). Para desde él comprender, a la vez que explicar, los complejos procesos político-jurídicos e institucionales de cambio social que se han producido a lo largo de casi trescientos años, circa 1750-circa 2020.

Es evidente que los objetos de estudio no pueden definir los períodos históricos; sin embargo, las definiciones que los historiadores damos a éstos implican el dominio historiográfico de una corriente o tendencia. Un hecho concreto, dada su trascendencia y significado: por ejemplo, la Revolución Francesa, la Revolución industrial, la guerra de la Independencia en España o las guerras carlistas, puede definir una etapa o caracterizar una época histórica. Si la categoría historiográfica mujer ha revisado y replanteado los significados del proceso histórico en el período de tiempo que hemos estudiado, el de familia ha ocupado dicha temporalidad por la amplitud de realidades que explica y refleja, tanto en procesos individuales de biografías y ciclos de vida como de redes de relación social. Se trata de incidir y profundizar en la reivindicación de los objetos como protagonistas explicativos del análisis histórico frente a la consideración de fragmentos y la superación de este estadio.

Llegados a este punto indicaría, a modo de conclusión final, el interés y la necesaria consideración de los siguientes puntos:

a) La fuerza del familismo en el conjunto de la sociedad española.

b) Integración de la evolución del proceso genealógico histórico en los siguientes apartados:

1.-Envejecimiento e incremento de los hogares unipersonales con procesos de gentrificación.

2.- Recuperación del papel del parentesco, subrayando la fuerza de los vínculos y lazos familiares para conocer mejor la naturaleza de las relaciones sociales, con análisis generacional e intergeneracional.

3.- Desacralización y desinstitucionalización en las relaciones y uniones personales.

c) Proseguir la integración en el conjunto social de las temáticas que ocupan y preocupan hoy al tercer sector, poniendo en relación la política de cuidados con lazos y vínculos de parentesco.

d) En perspectiva comparativa tener en cuenta la tendencia homogeneizadora en Europa, sin olvidar la diversidad regional de España. 
Bibliografía

ARIES, PH. (1948): Histoire des populations françaises et de leurs attitudes devant la vie depuis le XVIIlé siécle. Paris, editions Self.

BESTARD CAMPS, J. (1980): "La historia de la familia en el contexto de las ciencias sociales", Quaderns de I'Institut Catalá d'Antropologia, 2, pp. 154-162.

BESTARD CAMPS, J. (2001): "Antropología e Historia: algunas consideraciones en torno a la historia de la familia en Europa", en Santiago Castillo y Roberto Fernández (coords.), Historia Social y Ciencias Sociales. Lleida, Molino, pp. 85-100.

CASEY, J. (2003): "La invención de la comunidad y la historia social", Pedralbes, 23, pp. 779-796.

CHACÓN JIMÉNEZ, F. y HURTADO MARTÍNEZ, J. (1986): “Contribution à I'histoire de la famille dans les pays de la Mediterranée occidentale (1750-1850)", Annales de Démographie Historique, 2, pp. 155-182.

CHACÓN JIMÉNEZ, F. (1987): "Notas para el estudio de la familia en la región de Murcia durante el Antiguo Régimen", en J. Casey, F. Chacón et al., La familia en la España Mediterránea (siglos XV-XIX). Barcelona, Crítica, pp. 129-171.

CHACÓN JIMÉNEZ, F. (1991): "La historia de la familia desde la perspectiva de la historia social. Notas para nuevas propuestas de estudio", en Santiago Castillo (coord.), La historia social en España. Actualidad y perspectivas. Madrid, Siglo XXI, pp. 261-266.

CHACÓN JIMÉNEZ, F. (2003): "Pluralidad de los estudios sobre la familia", en A. Vera Estrada (comp.), La familia y las ciencias sociales, La Habana, Centro de Investigación y Desarrollo de la Cultura Cubana Juan Marinello, pp. 11-20.

CHACÓN JIMÉNEZ, F. (2009): “Familia y hogar en la sociedad española. Mitos y realidades históricas", en Francisco Javier Lorenzo Pinar (ed.), La Familia en la Historia. Salamanca, ediciones Universidad de Salamanca, pp. 121-134.

CHACÓN JIMÉNEZ, F. (2020): “Las familias como definidoras del proceso histórico, circa mitad siglo XVIII-siglo XXI. El ejemplo de España en el contexto europeo", en Enrique Pastor, Jorge Ferreira, Maria das Dores, Francisco Chacón, Familias, Identidades y cambio social en España y Portugal. Siglos XIX-XXI. Pamplona, Thompson Reuters, pp. 31-48.

CHACÓN MARTÍNEZ, A. (2019): El interés superior del menor. Historia de un reconocimiento jurídico en los derechos humanos para la infancia (siglos XVIII-XXI), Murcia, Editum. Ediciones de la Universidad de Murcia.

DELILLE, G. (2001): "Reflexions sur le système Européen de la parenté et de I'alliance, Annales, HSS, 2, pp. 369-380.

DELILLE, G. (2017): La economía de Dios. Familia y mercado entre cristianismo, judaísmo e islam. Madrid, ediciones Polifemo (traducción de 2013, L'economia di Dio. Famiglia e mercatotracristianesimo, ebraismo, islam. Roma, Salerno Editrice).

DOSSE, F. (1987): L'Histoire en miettes: des Annales à la «nouvelle histoire». Paris, La Decouverte.

DOUSSET, C.; FAGGCION, L.; MINVIELLE, S. y REGINA, C. (2013): "Parentalité: aproches historiques en Europe", Popolazones e Storia, S.I.D.E.S., pp. 9-15.

DOYON, J.; ODIER DA CRUZ, L.; PRAZ, A. F. y STEINBER, A. F. (2013): “Normes de parentalité: modelisation et regulations (XVIIIe-XXle siècles)", Annales de Démographie Historique. Dossier: Normes de parentalité, pp. 7-23.

GINZBURG, C. y PONI, C. (1979): "II nome e il come. Scambio ineguale e mercato storiográfico", QuaderniStorici, 40 (1), pp. 181-190 (traducción 1991, "El nombre y el cómo. Intercambio desigual y mercado historiográfico", Historia Social, 10, pp. 63-70).
HAREVEN, T. (1995): "Historia de la familia y la complejidad del cambio social”, Boletín de la ADEH, XIII, 1, pp. 99-149 (traducción 1991, “The History of the Family and the complexity of social change", American Historical Review, 96 (I), pp. 95-124).

HERNÁNDEZ FRANCO, J. (1996): Cultura y limpieza de sangre en la España moderna. Puritatesanguinis. Murcia, Editum.

HERNÁNDEZ FRANCO, J. (2011): Sangre limpia, sangre española. El debate de los estatutos de limpieza (siglos XV-XVII), Madrid, Cátedra.

LE GOFF, J. (2014): Faut-il vraiment decouper /'histoire en tranches?Paris, editions de Seuil.

PASTOR, E., FERREIRA, J., DAS DORES, M.D., CHACÓN, F. (2021): Familias, identidades y cambio social en España y Portugal. Siglos XIX-XXI. Perspectivas comparadas europeas. Pamplona, Thomson Reuters. Aranzadi.

PORQUERES, E. (2015): Individu, personne et parenté en Europe. París, editions Maison de Sciences de L'Homme (traducción 2021, colección Familia, élite de poder, Historia social, Murcia, Editum).

ROWLAND, R. (1983): “La metodología histórica de la «escuela de Cambridge» y su aplicación a las sociedades de Europa meridional", II Jornadas D'Estudis Historics Locals. Palma de Mallorca, Institut d'Estudis Balearics.

ROWLAND, R. (1989): "Población, Familia, Sociedad", Gestae. Taller de Historia, 1, Murcia.

TONNIES, F. (2009): Comunidad y asociación. El comunismo y el socialismo como formas de vida social, edición a cargo de José Luis Monereo Pérez. Granada, Comares.

WALL, R.; ROBIN, J. y LASLETT, P. (1983): Family Forms in Historic Europe. New York, Cambridge University Press.

WRIGLEY, E.A. y SCHOFIELD, R.S., (1981): The population History of England, 1541-1871. A Reconstruction. New York, Cambridge University Press. 